(C) 2017 IEEE. Personal use of this material is permitted. Permission from IEEE must be obtained for all other uses, in any current or future media, including reprinting/republishing this material for advertising or promotional purposes, creating new collective works, for resale or redistribution to servers or lists, or reuse of any copyrighted component of this work in other works. 


\title{
Capacity of Infrastructure-based Cooperative Vehicular Networks
}

\author{
Jieqiong Chen*, Guoqiang Mao*, Changle $\mathrm{Li}^{\dagger}$ \\ *School of Computing and Communications, University of Technology Sydney, NSW 2007, Australia \\ ${ }^{\dagger}$ State Key Laboratory of Integrated Services Networks, Xidian University, Xi'an, Shaanxi, 710071 China \\ Email: jieqiong.chen@student.uts.edu.au,g.mao@ieee.org, clli@mail.xidian.edu.cn
}

\begin{abstract}
In this paper, we propose a cooperative communication strategy that explores the combined use of vehicleto-infrastructure (V2I) communications, vehicle-to-vehicle (V2V) communications, mobility of vehicles and cooperation among vehicles and infrastructure to improve the achievable capacity of vehicular network. An analytical framework is developed to model the data dissemination process using this strategy, and a closed form expression of the achievable capacity is obtained, which reveals the relationship between the achievable capacity and its major performance-impacting parameters such as inter-infrastructure distance, radio ranges of infrastructure and vehicles, sensing range of vehicles, transmission rates of V2I and $\mathrm{V} 2 \mathrm{~V}$ communications, vehicular density and the proportion of vehicles with download requests. Numerical result shows that the proposed cooperative communication strategy significantly increases the capacity of vehicular networks, especially when the proportion of vehicles with download request is low. Our results provide guidance on the optimum deployment of vehicular network infrastructure and the design of cooperative communication strategy to maximize the capacity.
\end{abstract}

Index Terms-Data dissemination, cooperative communication, capacity, vehicular networks.

\section{INTRODUCTION}

Interest is surging on vehicular networks due to their increasingly important role in improving road traffic efficiency, enhancing road safety and providing real-time information to drivers and passengers [1]. Two wireless communication modes: vehicle-to-infrastructure (V2I) and vehicle-to-vehicle (V2V) communications are supported in vehicular networks by deploying wireless communication infrastructure points along the roadside (e.g., road-side units (RSU)), equipping vehicles with on-board communication facilities (e.g., onboard units (OBU)), and with assist of dedicated short-range communication (DSRC) [2] and LTE technology.

V2I and V2V communications, on one hand, are both fundamental techniques to disseminate data for vehicular applications, including safety applications like disseminating realtime information about traffic accidents, traffic congestion or obstacles in the road, and non-safety applications like offering value-added services (e.g., digital maps with real-time traffic status) and in-car entertainment services [1]; on the other hand, as pointed out in [3] and reference therein, purely relying on V2I communications or $\mathrm{V} 2 \mathrm{~V}$ communications alone cannot meet the diverse communication requirements of different vehicular applications. Instead, V2I and V2V communications have to co-exist and complement each other to improve the network performance.
In our prior work [3], we considered a vehicular network scenario where there is only one vehicle with download request from the Internet and all other vehicles cooperate to assist the communication of the aforementioned vehicle. We showed that a cooperative communication strategy, which utilizes both V2I and V2V communications, the mobility of vehicles and cooperation among vehicles and infrastructure, can significantly boost the throughput of the vehicle. In this paper, we extend to consider a more general scenario with a subset of vehicles, termed Vehicles of Interest (VoIs), having download requests. Each VoI downloads a distinct large-size file from the Internet and other vehicles without download requests, termed helpers, assist the delivery of the files to the VoIs. We analyze the capacity achievable by the VoIs using the cooperative communication strategy and develop an analytical framework to investigate the relationship between the achievable capacity and its major performance-impacting parameters. The novelty and major contributions of this paper are summarized as follows:

1) We propose a novel cooperative communication strategy, which utilizes V2I and V2V communications, mobility of vehicles, and cooperation among vehicles and infrastructure to boost capacity of vehicular networks;

2) We develop an analytical framework to model and investigate the data dissemination process assuming the aformentioned cooperative communication strategy, and a closed-form expression of the capacity achieved by the VoIs in a vehicular network is obtained;

3) Both simulations and numerical analysis are conducted, which show that the proposed cooperative strategy significantly improves the capacity of vehicular networks, compared with its non-cooperative counterpart, even when the proportion of VoIs is small.

Our results shed light on the optimum deployment of vehicular network infrastructure in terms of their interval distance, and the optimum design of cooperative communication strategy to improve the capacity of vehicular networks.

The rest of this paper is organized as follows: Section III introduces the system model, the proposed cooperative communication strategy and the problem formation. Theoretical analysis are provided in Section [II] In Section [V] we validate the analytical result using simulations and conduct numerical analysis to discuss our result and its insight. Section $\mathrm{V}$ concludes this paper. 


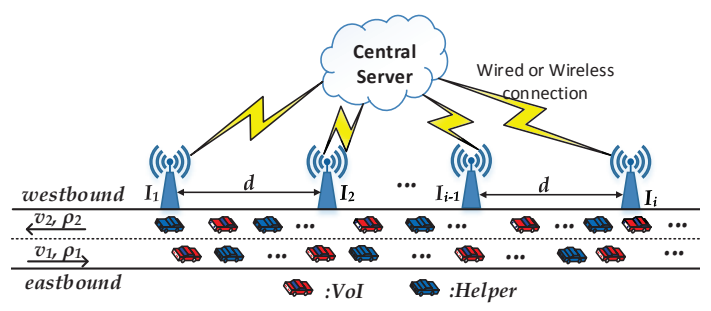

Fig. 1. An illustration of the vehicular network on a highway. The density and speed of vehicles in each direction are $\rho_{1}, v_{1}$ and $\rho_{2}, v_{2}$ respectively.

\section{System Model AND PROBLEM Formation}

\section{A. Network Model}

We consider a vehicular network on a bi-directional highway with length $L$ where roadside infrastructure are uniformly deployed with equal interval distance $d, d \ll L$. Considering that the width of a lane is typically small compared with the transmission range of vehicles, we ignore the road width and model multiple lanes in the same direction as one lane [4]. We further assume that all infrastructure points are connected to the Internet through wired or wireless backbone with much larger capacity than the vehicular networks.

We adopt a widely used traffic model that the distribution of vehicles in both directions follow Poisson processes with densities $\rho_{1}$ and $\rho_{2}$ respectively [5]-[7]. It follows that the inter-vehicle distances in each direction are exponentially distributed. Furthermore, as a ready consequence of the superposition property of Poisson processes [8], all vehicles on the highway are also Poissonly distributed with spatial density $\rho=\rho_{1}+\rho_{2}$. We assume that the proportion of VoIs in both directions are $p(0<p<1)$. Therefore, VoIs and helpers respectively have traffic density $p \rho$ and $(1-p) \rho$. Moreover, vehicles in both directions travel at a constant speed of $v_{1}$ and $v_{2}$ respectively. We will show later that our analysis also applies to other time-varying speed model, e.g., Gaussian speed model [9]. The system model is illustrated in Fig. 1

\section{B. Wireless Communication Model}

Both V2I and V2V communications are considered. All infrastructure points and vehicels are assumed to have the same radio range, denoted by $r_{I}$ and $r_{0}$ respectively. A pair of vehicles (or vehicle and infrastructure) can directly communicate with each other if and only if (iff) their Euclidean distance is not larger than the radio range $r_{0}\left(\right.$ or $r_{I}$ ) [4], [5], [10].

We consider that each vehicle has a single antenna so that they cannot transmit and receive at the same time. Besides, unicast scenario is considered that each infrastructure (or vehicle) can only transmit information to one vehicle at a time. We further assume that V2I and V2V communications are allocated different channels so that there is no mutual interference between them. For V2V communications, CSMA media access control (MAC) protocol is adopted with sensing range $R_{c}$. We assume $R_{c} \geq 2 r_{0}$ to avoid collision. Moreover, we assume V2I and V2V communicate at a constant data rate $w_{I}$ and $w_{V}$ respectively [11], [12]. For time-varying channels, the values of $w_{I}$ and $w_{V}$ can be replaced by the respective time-averaged data rate of $\mathrm{V} 2 \mathrm{I}$ and $\mathrm{V} 2 \mathrm{~V}$ communications and our analysis still applies.

We consider a prioritized V2I transmitting scheme that infrastructure will transmit its data to VoIs first. Helpers can receive data from the infrastructure only when there is no VoI within its coverage. For V2V communications, helpers function as transmitters and VoIs as receivers. A transmitter can choose a receiver from either direction within its transmission range and transmit data via one-hop communication.

\section{Cooperative Communication Strategy}

As mentioned previously, we consider a scenario where some VoIs (with proportion $0<p<1$ ) want to download large files, e.g., videos, from a remote server and the file to be downloaded by different VoI is different. Each requested large file by the VoIs may be first split into multiple pieces and transmitted to different infrastructure points such that each infrastructure point has a different piece of that file. Each piece of data delivered to infrastructure may be further split and transmitted either directly to the corresponding VoI requesting it or to helpers when they move into its coverage so that VoIs and helpers have different pieces of data. Each helper may store data for different VoIs. We assume there is a central server that has full knowledge of the data transmission process to guarantee that the data the helpers receive from infrastructure is the data required by the VoIs they will encounter. This assumption helps to establish the capacity (maximum data rate) that can be achieved. Therefore, when the VoIs are in the coverage of infrastructure, they receive data directly from the infrastructure. In the meantime, the helpers may also receive data from the infrastructure when they obtain access to the infrastructure. When the VoIs move outside the coverage of infrastructure, they may continue to receive data from helpers, exploiting the mobility of vehicles and V2V communications. In this way, V2I communications between the VoIs and infrastructure, between helpers and infrastructure, $\mathrm{V} 2 \mathrm{~V}$ communications between the VoIs and helpers, cooperations among infrastructure and among vehicles, as well as vehicular mobility are coherently combined to maximize the capacity of the VoIs.

\section{Problem Formation}

Consider an arbitrarily chosen time interval $[0, t]$ and denote the amount of data received by all VoIs as $D(t)$ during this time interval. In this paper, we are interested in finding the long-term achievable capacity of the VoIs using our cooperative communication strategy, where the long-term capacity, denoted by $\eta$, is formally defined as follows:

$$
\eta=\lim _{t \rightarrow \infty} \frac{D(t)}{t} .
$$

\section{THEORETICAL ANALYSIS}

We define the area covered by one infrastructure point (termed V2I Area) and the adjacent area between two consecutive infrastructure points but not covered by the infrastructure point (termed V2V Area) as a cycle, which has length $d$. See 


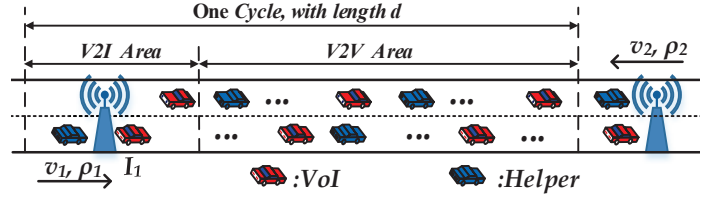

Fig. 2. An illustration of one cycle, which includes V2I Area and V2V Area.

Fig. 2 for an illustration. It follows from renewal theory [14] that the long-term achievable capacity by the VoIs from each cycle, denoted by $\eta_{\text {cycle, }}$, is identical and the total capacity achieved in a segment with length $L$ can be readily calculated by (ignoring the trivial fact that $\frac{L}{d}$ may not be an integer):

$$
\eta=\frac{L}{d} \eta_{\text {cycle }}
$$

From (2), to calculate the total capacity achievable by the VoIs from a highway segment with length $L$, it suffices to calculate the capacity achieved by the VoIs from one cycle, which can be calculated as follows:

$$
\eta_{\text {cycle }}=\lim _{t \rightarrow \infty} \frac{D_{V 2 I}(t)}{t}+\lim _{t \rightarrow \infty} \frac{D_{V 2 V}(t)}{t}
$$

where $D_{V 2 I}(t)$ and $D_{V 2 V}(t)$ are respectively the expected amount of data received by the VoIs from infrastructure in the V2I Area and from helpers in the V2V Area during time period $t$. In the following, we will focus on studying one cycle entirely contained within the highway segment of length $L$, termed the cycle of interest and calculate the two terms on the right hand side of (3) separately.

\section{A. Capacity achieved by VoIs from V2I communications}

Without loss of generality, we term the infrastructure point located in our cycle of interest $I_{1}$. We assume that time is divided into time slot with equal length $\Delta t$, and $\Delta t$ is rather small that we can regard vehicles as static during each time slot. Denote by $q_{1}(i), i=1,2, \ldots$ a discrete random variable representing the fraction of time that VoIs' V2I communication happen during the $i$ th time interval. Recall that in our assumption, infrastructure delivers its data directly to the VoIs as long as there are VoIs within its coverage. Therefore, $q_{1}(i)$ equals to 1 when there exist at least one VoI within the coverage of $I_{1}$ during the $i$ th time interval, otherwise equals to 0 . It follows that the expected total amount of data the VoIs can obtain through V2I communications during time period $[0, t]$ can be calculated by (ignoring the trivial fact that $\frac{t}{\Delta t}$ may not be an integer):

$$
D_{V 2 I}(t)=\lim _{\triangle t \rightarrow 0} E\left(w_{I} \sum_{i=1}^{t / \Delta t} q_{1}(i) \triangle t\right) .
$$

According to the ergodicity and stationarity properties of homogeneous Poisson point process [15], the time average of $q_{1}(i), \lim _{t \rightarrow \infty} \frac{\lim _{\triangle t \rightarrow 0} E\left(\sum_{i=1}^{t / \Delta t} q_{1}(i) \triangle t\right)}{t}$, is equal to the probability that there is at least one VoI within the coverage of $I_{1}$ at a randomly chosen time slot, denoted by $\bar{q}_{1}$. From the Poisson distribution of vehicles, $\bar{q}_{1}=1-e^{-p \rho 2 r_{I}}$. Therefore,

$$
\lim _{t \rightarrow \infty} \frac{\lim _{\triangle t \rightarrow 0} E\left(\sum_{i=1}^{t / \triangle t} q_{1}(i) \triangle t\right)}{t}=\bar{q}_{1}=1-e^{-p \rho 2 r_{I}} .
$$

Combing (4)-(5), we have:

$$
\lim _{t \rightarrow \infty} \frac{D_{V 2 I}(t)}{t}=w_{I}\left(1-e^{-p \rho 2 r_{I}}\right)
$$

\section{B. Capacity achieved by VoIs from V2V communications}

Note that the data received by the VoIs from helpers eventually comes from the data received by the helpers from infrastructure. Therefore, the amount of data the VoIs can receive from $\mathrm{V} 2 \mathrm{~V}$ communications during time period $[0, t]$, on one hand, is constrained by how much data the helpers can receive via their V2I communications during time period $[0, t]$; on the other hand, is limited by how much data the helpers can transmit to the VoIs through V2V communications during time period $[0, t]$. Taking the above two constraints into account, we have the following results:

Theorem 1. The capacity the VoIs can achieve through V2V communications from one cycle is given by:

$$
\lim _{t \rightarrow \infty} \frac{D_{V 2 V}(t)}{t}=\min \left\{\lim _{t \rightarrow \infty} \frac{D_{I_{-} H}(t)}{t}, \lim _{t \rightarrow \infty} \frac{D_{V}(t)}{t}\right\},
$$

where $D_{I_{-}}(t)$ is the expected amount of data received by helpers from one cycle through their V2I communications during time period $[0, t]$, and $D_{V}(t)$ is the expected amount of data the helpers can deliver to the VoIs through $V 2 V$ communications in the V2V Area during time period $[0, t]$ without considering the limitation of the amount of data received by helpers from the infrastructure.

Since the bottleneck is either in the $\mathrm{V} 2 \mathrm{~V}$ communications between VoIs and helpers, or in the V2I communications between helpers and the infrastructure, the proof of Theorem 1 follows readily.

From Theorem 11, it remains to calculate $\lim _{t \rightarrow \infty} \frac{D_{I_{-} H}(t)}{t}$ and $\lim _{t \rightarrow \infty} \frac{D_{V}(t)}{t}$. In the following, we will calculate these two terms separately.

1) Calculation of $\lim _{t \rightarrow \infty} \frac{D_{I_{-} H}(t)}{t}$ : Denote by $q_{2}(i)$ a discrete random variable, which equals to 1 when helper's V2I communication happens during $i$ th time interval, otherwise equals to 0 . Similar to the analysis in $\amalg$ II-A we have

$$
\begin{aligned}
\lim _{t \rightarrow \infty} \frac{D_{I_{-} H}(t)}{t} & =\lim _{t \rightarrow \infty} \frac{w_{I} \lim _{\triangle t \rightarrow 0} E\left(\sum_{i=1}^{t / \triangle t} q_{2}(i) \triangle t\right)}{t} \\
& =w_{I} \bar{q}_{2},
\end{aligned}
$$

where $\bar{q}_{2}$ is the probability that helper's V2I communication happens at a randomly chosen time instant. Note that an infrastructure point only delivers its data to helpers if both of the following conditions are met: (i) there is no VoI within its coverage, and (ii) there is at least one helper within its coverage. Thus, $\bar{q}_{2}$ can be readily calculated by:

$$
\bar{q}_{2}=e^{-p \rho 2 r_{I}} \cdot\left(1-e^{-(1-p) \rho 2 r_{I}}\right)=e^{-p \rho 2 r_{I}}-e^{-\rho 2 r_{I}} .
$$


Combing (8) and (9), we have:

$$
\lim _{t \rightarrow \infty} \frac{D_{I_{-} H}(t)}{t}=w_{I}\left(e^{-p \rho 2 r_{I}}-e^{-\rho 2 r_{I}}\right) .
$$

2) Calculation of $\lim _{t \rightarrow \infty} \frac{D_{V}(t)}{t}$ : Recall that for $\mathrm{V} 2 \mathrm{~V}$ communications, we adopt CSMA multiple access protocol with sensing range $R_{c}$. Therefore, a helper within the V2V Area can potentially be chosen as one of the simultaneously transmitters when there is no other helper transmitting within its sensing range and there is at least one VoIs within its transmission range. We call a helper, together with the VoI that the helper transmits to, an active helper-VoI pair iff this helper is chosen as a transmitter and chooses this VoI within its transmission range as its receiver.

Denote by $N_{p}^{\chi}(i)$ the number of active helper-VoI pairs in the V2V Area during $i$-th time slot, where $\chi \in \Phi$ denotes the scheduling algorithm that schedules the simultaneously active helper-VoI pairs and $\Phi$ denotes the set of all scheduling algorithms. Using the analysis in [12], [13], we have:

$$
\begin{aligned}
\lim _{t \rightarrow \infty} \frac{D_{V}^{\chi}(t)}{t} & =\lim _{t \rightarrow \infty} \frac{w_{V} \lim _{\triangle t \rightarrow 0} E\left(\sum_{i=1}^{t / \Delta t} N_{p}^{\chi}(i) \triangle t\right)}{t} \\
& =w_{V} E\left[N_{p}^{\chi}\right],
\end{aligned}
$$

where $D_{V}^{\chi}(t)$ is the respective $D_{V}(t)$ assuming the scheduling scheme $\chi$, and $E\left[N_{p}^{\chi}\right]$ is the expected number of simultaneously active helper-VoI pairs in the $\mathrm{V} 2 \mathrm{~V}$ Area at a randomly chosen time slot.

From (11), the maximum value of $\lim _{t \rightarrow \infty} \frac{D_{V}^{\chi}(t)}{t}$ is achieved when using an optimum scheduling that schedules as many active helper-VoI pairs as possible. Therefore, in the following analysis, we will first find an optimum scheduling scheme, denoted by $\chi_{\text {opt }}$, that leads to the maximum number of simultaneously active helper-VoI pairs, and then calculate $E\left[N_{p}^{\chi_{o p t}}\right]$ under this optimum algorithm. Without loss of generality, we designate the left boundary point of the V2V Area, i.e., the point to the right of infrastructure point $I_{1}$ and at a distance $r_{I}$ to $I_{1}$, as the origin of the coordinate system, and the right direction as positive $(+x)$ direction. The following theorem summarizes the optimum scheduling scheme.

Theorem 2. An optimum scheduling scheme $\chi_{o p t}$, which leads to the maximum number of simultaneously active helper-VoI pairs in V2V Area is as follows: select active helper-VoI pairs in order from left to the right. First, choose the first helper to the right of the origin that has at least one VoIs within its coverage as the first transmitter, and the left-most VoI within the coverage of that helper as its receiver. The next transmitter is the nearest helper to the current transmitter, and satisfies the following conditions: 1) the distance between this helper and the current transmitter is larger than $\left.R_{c} ; 2\right)$ it can find at least one VoI within its coverage. If there are multiple VoIs, always chooses the leftmost VoI. Repeat the above process until the rightmost border of the V2V Area is reached.

Proof: Denote by $X_{k}, k=1,2, \ldots$ the location of the $i$ th transmitter (helper of the active helper-VoI pair), numbered from left to the right, under the optimum scheduling scheme $\chi_{o p t}$. Denote by $Y_{k}, k=1,2, \ldots$ the location of the $i$ th transmitter under an arbitrary scheduling scheme $\chi^{\prime}$. It

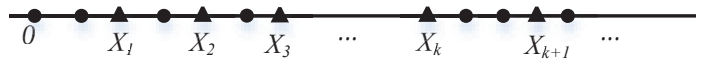

(a) Results under the scheduling scheme $\chi_{\text {opt }}$

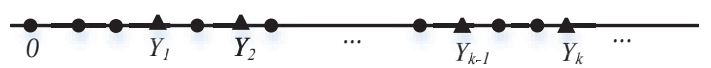

(b) Results under another scheduling scheme $\chi^{\prime}$

Fig. 3. An illustration of the distribution of simultaneous transmitters, where the triangular points and dots represent the helpers that are and are not chosen as simultaneous transmitters respectively.

follows that $X_{1}<X_{2}<\cdots<X_{k}<X_{k+1}<\cdots$ and $Y_{1}<Y_{2}<\cdots<Y_{k}<Y_{k+1}<\cdots$. See Fig. 3 for an illustration. In the following, we prove that $\chi_{o p t}$ described in Theorem 2 is an optimum scheduling scheme that would lead to the maximum number of active helper-VoI pairs by recursion that $X_{k} \leq Y_{k}$ holds for any $k=1,2, \ldots$.

For $k=1$, noting that according to the scheduling scheme $\chi_{\text {opt }}$, the first transmitter is the leftmost helper in the V2V Area that has at least one VoI within its coverage. Therefore, it follows readily that $X_{1} \leq Y_{1}$.

Assuming that $X_{k} \leq Y_{k}$ when $k=n, n \geq 1$, we will show that $X_{n+1} \leq Y_{n+1}$. We consider two different cases: $X_{n+1} \leq Y_{n}$ and $X_{n+1}>Y_{n}$ :

(i) Case $X_{n+1} \leq Y_{n}$ : in this case, it can be readily shown that $X_{n+1} \leq Y_{n}<Y_{n+1}$.

(ii) Case $X_{n+1}>Y_{n}$ : in this case, under the scheduling scheme $\chi_{\text {opt }}$, the $(n+1)$-th transmitter is the nearest helper to the right of the $n$-th transmitter satisfying simultaneous transmission conditions: it is outside the sensing range of the $n$-th transmitter who are located at $X_{n}$ and has at least one VoI within its transmission range. Therefore, there is no helper within road segment $\left(X_{n}, X_{n+1}\right)$ that can transmit simultaneously. If $Y_{n+1}<X_{n+1}$, a contradiction must occur. Thus, $X_{n+1} \leq Y_{n+1}$.

Therefore, $X_{k} \leq Y_{k}$ holds for any $k=1,2, \ldots$ It readily follows that the number of simultaneously active helper-VoI pairs under $\chi^{\prime}$ must be less than or equal to that under $\chi_{o p t}$, which finalize the proof.

Now we calculate the expected maximum number of active help-VoI pairs, $E\left[N_{P}^{\chi_{o p t}}\right]$, and the corresponding value of $\lim _{t \rightarrow \infty} \frac{D_{V}(t)}{t}$ under the optimum scheme $\chi_{\text {opt }}$.

Denote by $S_{k} \in\left[0, d-2 r_{I}\right], k=1,2, \ldots$ the position of the $k$-th transmitter (helper in the active helper-VoI pair) under the optimum scheduling scheme $\chi_{\text {opt }}$. Denote by $L_{k}, k=1,2, \ldots$ the distance between the $k$-th and the $(k+1)$-th transmitter, and $L_{0}$ the distance between the first transmitter and the origin. Denote by $V_{k, 1}$ the first helper located within road segment $\left[S_{k}+R_{c}, d-2 r_{I}\right]$, by $V_{k, 2}$ the second helper, and so on. Denote by $l_{k, i}, i=1,2, \ldots$ the distance between helper $V_{k, i}$ and $V_{k, i+1}$ and by $l_{k, 0}$ the distance between helper $V_{k, 1}$ and the point $S_{k}+R_{c}$. See Fig. 目 for an illustration.

Suppose the $(k+1)$ th transmitter is exactly the $m_{k}$-th helper located within road segment $\left[S_{k}+R_{c}, d-2 r_{I}\right]$, then we have $L_{k}=R_{c}+\sum_{i=0}^{m_{k}-1} l_{k, i}$, where $m_{k}$ is a random integer. According to the scheduling scheme $\chi_{o p t}$, the $(k+1)$ th transmitter is the first helper within road segment $\left[S_{k}+R_{c}, d-2 r_{I}\right]$ 


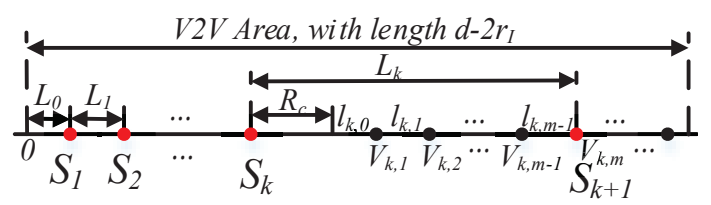

Fig. 4. An illustration of the distribution between two consecutive simultaneous transmitters.

that can find a VoI within its coverage, therefore, we have $\operatorname{Pr}\left(m_{k}=1\right)=1-e^{-p \rho 2 r_{0}}$ and when $m \geq 2$,

$$
\begin{aligned}
& \operatorname{Pr}\left(m_{k}=m\right) \\
= & \operatorname{Pr}\left(\text { no VoI within the coverage of } V_{k, i}, i=1, \ldots m-1\right. \\
& \left.\cap \exists \text { VoI within the coverage of } V_{k, m}\right) \\
= & \operatorname{Pr}\left(\text { no VoI in } \sum_{i=1}^{m-2} \min \left\{l_{k, i}, 2 r_{0}\right\}+2 r_{0}\right) \\
& \times \operatorname{Pr}\left(\text { there exists VoI in } \min \left\{l_{k, m-1}, 2 r_{0}\right\}\right) \\
= & E\left[e^{-p \rho\left(\sum_{i=1}^{m-2} \min \left\{l_{k, i}, 2 r_{0}\right\}+2 r_{0}\right)}\right] \\
& \times\left(1-E\left[e^{-p \rho\left(\min \left\{l_{k, m-1}, 2 r_{0}\right\}\right)}\right]\right) \\
= & e^{-p \rho 2 r_{0}} \cdot c_{1}^{m-2}\left(1-c_{1}\right),
\end{aligned}
$$

where $c_{1}=1-p+p e^{-\rho 2 r_{0}}$. The second step results due to the property that VoIs have a Poisson distribution and therefore the VoIs in non-overlapping intervals are independent. The third step is a result from the Poisson distribution of the VoIs and a use of total probability theorem.

Noting that $L_{k}, k=1,2, \ldots$ are i.i.d and are also independent of $L_{0}$, therefore, $E\left[N_{p}^{\chi_{o p t}}\right]$ can be regarded as the expected number of renewals of a delayed renewal counting process in a road segment with length $d-2 r_{I}$, with delay $E\left[L_{0}\right]$ and each renewal having an average length $E\left[L_{k}\right], k=1,2, \ldots$. Considering that $d$ is much larger compared to the distance between two consecutive simultaneous transmitters, therefore, $E\left[N_{p}^{\chi_{o p t}}\right]$ can be approximately calculated according to the Elementary Delayed Renewal Theorem [14, Theorem 5.8.4]:

$$
E\left[N_{p}^{\chi_{o p t}}\right]=\frac{d-2 r_{I}}{E\left[L_{k}\right]} .
$$

Noting that each $l_{k, i}, i=1,2, \ldots m_{k}-1$ follows identical and independent exponential distribution with mean value $\frac{1}{(1-p) \rho}$, and due to the memoryless property of exponential distribution, $l_{k, 0}$ also has the same distribution as $l_{k, i}, i=$ $1,2, \ldots m_{k}-1$. This follows that:

$$
\begin{aligned}
E\left[L_{k}\right] & =E\left[R_{c}+\sum_{i=0}^{m_{k}-1} l_{k, i}\right] \\
& =R_{c}+\sum_{m=1}^{\infty} m \cdot E\left[l_{k, i}\right] \operatorname{Pr}\left(m_{k}=m\right) \\
& =R_{c}+\sum_{m=1}^{\infty} \frac{m}{(1-p) \rho} \operatorname{Pr}\left(m_{k}=m\right) \\
& =R_{c}+\frac{p-p e^{-\rho 2 r_{0}}+e^{-p \rho 2 r_{0}}}{(1-p) \rho\left(1-e^{-\rho 2 r_{0}}\right)} .
\end{aligned}
$$

Combining (11), (13) and (14), we have:

$$
\begin{aligned}
& \lim _{t \rightarrow \infty} \frac{D_{V}(t)}{t} \\
= & \frac{w_{V}(1-p) p \rho\left(1-e^{-\rho 2 r_{0}}\right)\left(d-2 r_{I}\right)}{(1-p) p \rho\left(1-e^{-\rho 2 r_{0}}\right) R_{c}+p-p e^{-\rho 2 r_{0}}+e^{-p \rho 2 r_{0}}} .
\end{aligned}
$$

\section{Achievable capacity}

Combining the analysis results shown in Section $\amalg I-A$ and III-B the total capacity achieved by the VoIs from a highway segment with length $L$ can be readily obtained:

$$
\begin{aligned}
\eta= & \frac{L}{d} \times \lim _{t \rightarrow \infty} \frac{D_{V 2 I}(t)+D_{V 2 V}(t)}{t} \\
= & \frac{L}{d} \times \min \left\{w_{I}\left(1-e^{-\rho 2 r_{I}}\right),\right. \\
& \left.w_{I}\left(1-e^{-p \rho 2 r_{I}}\right)+\frac{w_{V} c\left(d-2 r_{I}\right)}{c R_{c}+p-p e^{-\rho 2 r_{0}}+e^{-p \rho 2 r_{0}}}\right\},
\end{aligned}
$$

where $c=(1-p) p \rho\left(1-e^{-\rho 2 r_{0}}\right)$.

Remark 3. It is interesting to note from (16) that the achievable capacity is irrelevant to the velocity of vehicles, which appears to be counter-intuitive at the first sight. This can be explained from the data dissemination process. As (6) and (11) show, both the capacity achieved by the VoIs from infrastructure and from helpers only depend on the spatial distribution of vehicles. In our system, the vehicles' arrival follows a Poisson process and the vehicles move at a constant speed. Therefore, the spatial distribution of the vehicles are both stationary and ergodic [15]. It follows that the capacity that can be achieved by the VoIs is independent of vehicular velocities. This observation implies that when vehicles arrive following a Poisson process, our analysis assuming the constant speed model is also applicable to other time-varying speed model, e.g., Gaussian speed model, as long as the resulting spatial distribution of vehicles is time-invariant, i.e., stationary.

\section{Simulation AND Discussion}

In this section we conduct Monte-Carlo simulations to establish the accuracy of our theoretical result and discuss its insights. Specifically, we set the length of a highway segment $L=100 \mathrm{~km}$. Eastbound and westbound vehicles move at constant speed $v_{1}=20 \mathrm{~m} / \mathrm{s}$ and $v_{2}=25 \mathrm{~m} / \mathrm{s}$ respectively. The radio range of infrastructure points and vehicles are $400 \mathrm{~m}$ and $200 \mathrm{~m}$, and all the vehicles have the same sensing range $R_{c}=400 \mathrm{~m}$. The data rate of V2I and V2V communications are $w_{I}=20 \mathrm{Mb} / \mathrm{s}$ and $w_{V}=2 \mathrm{Mb} / \mathrm{s}$ respectively. Each simulation is repeated 2000 times and the average value is shown in the plot.

Fig. 5] demonstrates a comparison of the capacity achieved by VoIs from one cycle and shows that the analytical results match very well with the simulations. Moreover, it also reveals the relationship between the capacity achieved from one cycle and the proportion of VoIs $p$, and shows that the capacity increases to its maximum value when the proportion of VoIs is larger than a threshold $p_{t h}$. Beyond that threshold, a further increase in $p$ has little impact on the capacity. This can be 


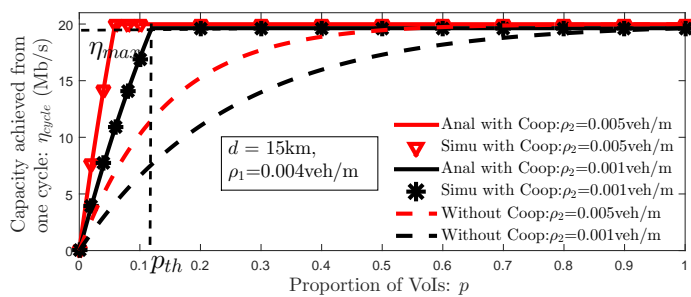

Fig. 5. A comparison of the capacity achieved by VoIs from one cycle between simulation and analysis, and a comparison between the capacity achieved with and without cooperative communication.

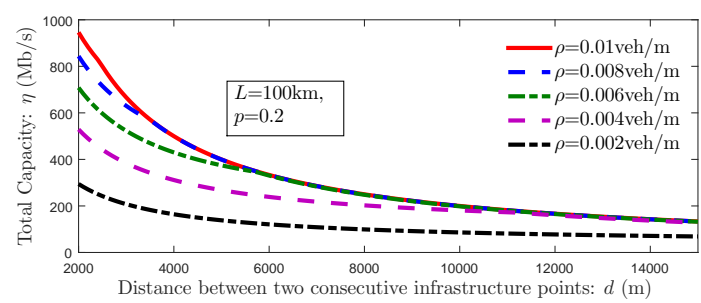

Fig. 6. Relationship between achievable capacity and inter-infrastructure distance.

explained by that when $p<p_{t h}$, the number of VoIs is insufficient to retrieve all the data received by the helpers from their V2I communications. Therefore, an increase in $p$ would significantly increase the number of simultaneous active helper-VoI pairs and consequently boost the capacity. However, when the proportion of VoIs reaches a certain threshold, VoIs can retrieve almost all the data received by the helpers from their V2I communications. In this case, the capacity achieved by the VoIs from one cycle approaches its maximum value $\eta_{\max }=w_{I}\left(1-e^{-\rho 2 r_{I}}\right)$, which is equal to the average data rate the infrastructure point delivers its data to all vehicles, including both VoIs and helpers.

Fig. 5] also compares the capacity from one cycle using our cooperative communication strategy (labeled as with Coop) with its non-cooperative counterpart (labeled as without Coop), and shows that our cooperative communication strategy can improve the capacity, even when there is only a small number of vehicles with download requests, i.e., a small $p$. The result for the non-cooperative counterpart is obtained by letting the VoIs only receive data from infrastructure. Furthermore, we can see that under the same network setting, without using the cooperative communication strategy, only when all vehicles have download requests, i.e., $p=1$, the maximum capacity $\eta_{\max }=w_{I}\left(1-e^{-\rho 2 r_{I}}\right)$ can be achieved. In contrast, with the cooperative communication strategy, this maximum capacity $\eta_{\max }$ can be achieved even when a small proportion of vehicles have download requests. This validates the effectiveness of cooperative communications to boost network performance.

Fig. 6 demonstrates the relationship between the capacity and the inter-infrastructure distance, and gives insight into the optimum vehicular network infrastructure deployment in terms of their interval distance under different traffic density. It is shown that the total capacity decreases with an increase of $d$. Furthermore, it can be seen that to achieve the same capacity, when the vehicular density is lower, the inter-infrastructure distance needs to be smaller. Therefore, when determining the optimum deployment of vehicular network infrastructure, it is important to take the vehicular density into account, e.g., in areas where the vehicular density is usually large, by utilizing a cooperative communication strategy, the number of infrastructure points can be reduced.

\section{Conclusions}

In this paper, we analyzed the capacity of vehicular networks with a finite traffic density adopting a cooperative communication strategy, which utilizes V2I communications, $\mathrm{V} 2 \mathrm{~V}$ communications, mobility of vehicles, and cooperation among vehicles and infrastructure to facilitate the transmission. A closed-form expression of the achievable capacity was obtained. Our result showed that the proposed cooperative strategy can improve the capacity of vehicular networks, and the improvement is more pronounced when the proportion of vehicles with download request is low. Moreover, our result sheds insight into the optimum deployment of vehicular network infrastructure and the design of cooperative communication strategy to maximize the capacity.

\section{ACKNOWLEDGMENT}

This research is supported by the Chinese National Natural Science Foundation project 61571350 and 61401334, Key Research and Development Program of Shaanxi Province, China (Contract No.2017KW-004, 2017ZDXM-GY-022).

\section{REFERENCES}

[1] S. Ilarri, T. Delot, R. Trillo-Lado, "A Data Management Perspective on Vehicular Networks," IEEE Commun. Surveys Tuts., vol. 17, no. 4, pp. 2420-2460, Fourth Quarter, 2015.

[2] J. B. Kenney, "Dedicated Short-Range Communications (DSRC) Standards in the United States," Proceedings of the IEEE, vol. 99, no. 7, pp. 1162-1182, 2011.

[3] J. Chen, G. Mao et al., "Throughput of Infrastructure-Based Cooperative Vehicular Networks", accepted by IEEE Trans. Intell. Transp. Syst. 2017.

[4] W. Zhang, et al., "Multi-Hop Connectivity Probability in InfrastructureBased Vehicular Networks," IEEE J. Sel. Areas Commun., vol. 30, no. 4, pp. 740-747, May. 2012.

[5] A. B. Reis, et al., "Deploying Roadside Units in Sparse Vehicular Networks: What Really Works and What Does Not," IEEE Trans. Veh. Technol., vol. 63, no. 6, pp. 2794-2806, Jul. 2014.

[6] G. Mao and B. Anderson, "Connectivity of Large Wireless Networks under a General Connection Model”, IEEE Trans. Inf. Theory, vol. 59, no. 3, pp. 1761 - 1772, 2013.

[7] X. Ge, et al., "5G Ultra-Dense Cellular Networks", IEEE Wireless Commun.,vol. 23, no. 1, pp. 72-79, 2016.

[8] R. Nelson, Probability, Stochastic Processes, and Queueing Theory: The Mathematics of Computer Performance Modeling. New York: SpringerVerlag, 1995.

[9] Z. Zhang, et al., "Stochastic Characterization of Information Propagation Process in Vehicular Ad hoc Networks," IEEE Trans. Intell. Transp. Syst., vol. 15, no. 1, pp. 122-135, Feb. 2014.

[10] G. Mao, "Connectivity of Communication Networks", Springer, ISBN 978-3-319-52989-9, March 2017.

[11] P. Gupta and P. Kumar, "The capacity of wireless networks," IEEE Trans. Inf. Theory, vol. 46, no. 2, pp. 388-404, 2000.

[12] G. Mao, et al., "Towards a Simple Relationship to Estimate the Capacity of Static and Mobile Wireless Networks," IEEE Trans. Wireless Commun., vol. 12, no. 9, pp. 3883-3895, 2013.

[13] G. Mao and B. Anderson, "Capacity of Large Wireless Networks with Generally Distributed Nodes", IEEE Trans. Wireless Commun., vol. 13 , no. 3, pp. 1678 - 1691, 2014.

[14] R. G. Gallager, Stochastic Processes: Theory for Applications. Cambridge University Press, 2013.

[15] F. Baccelli and B. Błaszczyszyn, Stochastic Geometry and Wireless Networks Volume II Applications, 2009. 